Article

\title{
Cloning and Expression Analysis of the BocMBF1c Gene Involved in Heat Tolerance in Chinese Kale
}

\author{
Lifang Zou ${ }^{1,2}$, Bingwei Yu ${ }^{1}$, Xing-Liang Ma ${ }^{2}$, Bihao Cao ${ }^{1}$, Guoju Chen ${ }^{1}$, Changming Chen ${ }^{1}$ \\ and Jianjun Lei ${ }^{1,3, *}$ \\ 1 Key Laboratory of Horticultural Crop Biology and Germplasm Innovation in South China, Ministry of \\ Agriculture, College of Horticulture, South China Agricultural University, Guangzhou 510642, China; \\ lifang.zou@gifs.ca (L.Z.); yubingwei@outlook.com (B.Y.); caobh01@scau.edu.cn (B.C.); \\ gjchen@scau.edu.cn (G.C.); cmchen@scau.edu.cn (C.C.) \\ 2 Seed and Developmental Biology Program, Global Institute for Food Security, University of Saskatchewan, \\ Saskatoon, SK S7N 0W9, Canada; Xingliang.ma@gifs.ca \\ 3 Henry School of Agricultural Science and Engineering, Shaoguan University, Shaoguan 512005, China \\ * Correspondence: jjlei@scau.edu.cn; Tel.: +86-133-8005-5679
}

Received: 3 September 2019; Accepted: 8 November 2019; Published: 11 November 2019

check for updates

\begin{abstract}
Chinese kale (Brassica oleracea var. chinensis Lei) is an important vegetable crop in South China, valued for its nutritional content and taste. Nonetheless, the thermal tolerance of Chinese kale still needs improvement. Molecular characterization of Chinese kale's heat stress response could provide a timely solution for developing a thermally tolerant Chinese kale variety. Here, we report the cloning of multi-protein bridging factor $(M B F) 1 c$ from Chinese kale (BocMBF1c), an ortholog to the key heat stress responsive gene MBF1c. Phylogenetic analysis showed that BocMBF1c is highly similar to the stress-response transcriptional coactivator $M B F 1 c$ from Arabidopsis thaliana $(A t M B F 1 c)$, and the BocMBF1c coding region conserves MBF1 and helix-turn-helix (HTH) domains. Moreover, the promoter region of BocMBF1c contains three heat shock elements (HSEs) and, thus, is highly responsive to heat treatment. This was verified in Nicotiana benthamiana leaf tissue using a green fluorescent protein (GFP) reporter. In addition, the expression of BocMBF1c can be induced by various abiotic stresses in Chinese kale which indicates the involvement of stress responses. The BocMBF1c-eGFP (enhanced green fluorescent protein) chimeric protein quickly translocated into the nucleus under high temperature treatment in Nicotiana benthamiana leaf tissue. Overexpression of BocMBF1c in Arabidopsis thaliana results in a larger size and enhanced thermal tolerance compared with the wild type. Our results provide valuable insight for the role of BocMBF1c during heat stress in Chinese kale.
\end{abstract}

Keywords: Chinese kale; thermal tolerance; BocMBF1c; expression; localization

\section{Introduction}

Chinese kale (Brassica oleracea var. chinensis Lei Syn:Brassica alboglabra Bailey) is a popular vegetable for its health benefits and taste [1]. Chinese kale, belonging to the Brassica genus, is native to China and, currently, is predominantly cultivated in South China and Southeast Asia [1]. Recently, the importance of Chinese kale has generated interest in its nutrition and genetics [2]. In particular, Chinese kale contains high levels of glucoraphanin which may possess cancer prevention properties [3].

Unfortunately, Chinese kale is very sensitive to heat stress, which often leads to decreased yield and quality [4]. Consequently, the main growing season and distribution of Chinese kale is limited to the winter season of South China.

High temperature can disturb the membrane, structure of protein, and chromatin architecture of the plant cells which respond with several signaling pathways to address the stress [5]. Many 
plant hormones are reported in the heat response of plants, and exogenous application of plant hormones can activate expression of heat stress-related genes thus improving thermal tolerance. For example, applying abscisic acid (ABA) can increase the thermal tolerance level by upregulating $A B A$ responsive transcription factors ( $A B R F s$ ), heat stress transcription factor (HSF) A2c (FaHSFA2c), and heat shock proteins (HSPs) in tall fescue [6]; treatment with methyl jasmonate (MeJA) stimulates the expression of HSP70 [7]; and exogenous salicylic acid (SA) increases the heat stress resistance of wheat by regulating the accumulation of ethylene (ET) and proline, increasing the efficiency of nitrogen usage [8]. Application of ET and jasmonic acid (JA) can activate thermal stress-related genes by upregulating ethylene response factor (ERF) 1 in Arabidopsis.

In plants, enormous progress has recently been made in understanding of the molecular mechanism of thermal tolerance [9]. In addition to HSF- and HSP-responsive pathways [9], the transcriptional coactivator Multi-protein bridging factor1c (MBF1c) was identified as a critical regulator for thermal tolerance responses in Arabidopsis [10]. In Arabidopsis, heat stress can lead to increased expression of $A t M B F 1 c$ and triggers the nuclear localization of AtMBF1c proteins. Nuclear AtMBF1c then function as transcription factors to regulate the downstream SA, trehalose, and ET thermal resistance-related pathways [10]. The AtMBF1c is a trans-acting regulatory element which recognizes CTAGA as a potential binding sequence and can regulate 36 genes, including stress-responsive gene dehydration-responsive element (DRE)-binding protein $2 A$ (DREB2A) [11]. In addition, overexpression of AtMBF1c and its ortholog from wheat can improve the performance of host plants under high temperature [11,12]. Therefore, $M B F 1 c$ is an ideal gene for improving plant thermal tolerance and, thus, increase productivity under heat stress. The expression of the MBF1c gene in Antarctic Moss (PaMBF1c) can be induced by several abiotic stresses, and overexpression of PaMBF1c can enhance heat, cold, and salinity stress tolerance in Arabidopsis [12]. However, to date there have been no studies conducted on the MBF1c of Chinese kale. In this study, we reported the ortholog of AtMBF1c in Chinese kale, termed BocMBF1c, and characterized its potential role in regulating heat tolerance in Chinese kale.

\section{Results}

\subsection{Cloning and Analysis of the BocMBF1c Gene}

As the genome sequence of Chinese kale is still unavailable, we used the Brassica rapa MBF1c gene (LOC103828628) as a reference sequence for primer design and then cloned the coding region of MBF1c from Chinese kale (variety "Ai-jiao-xiang-gu") genomic DNA. The resultant amplicon of $707 \mathrm{bp}$ was sequenced and confirmed to be BocMBF1c by sequence homology analysis (Figure 1a,b). Open reading fragment (ORF) prediction by ORF finder did not find any intron within the BocMBF1c gene, which was then confirmed by agarose gel analysis (Figure S1). Amino acid sequence analysis identified multi-protein bridging factor1 (MBF1) and helix-turn-helix (HTH) domains (Figure 1a). Phylogenetic analysis indicated that BocMBF1c clustered with Arabidopsis MBF1c (Figure 1b, Table S1). Next, we obtained the MBF1c gene and promoter region of Brassica oleracea (Bol013952) by searching the BocMBF1c coding sequence (CDS) using BLASTN (http://brassicadb.org/brad/blastPage.php). The $2005 \mathrm{bp}$ DNA fragment containing the promoter of BocMBF1c was cloned with primers designed from $B O M B F 1 c$, from which we used 1409 bp upstream sequence from initiation codon for further analysis. The BocMBF1c gene was submitted to GenBank (Accession number: MH685643).

Sequence analysis of the $1409 \mathrm{bp}$ BocMBF1c promoter revealed multiple stress and hormonal responsive cis-elements, including heat shock elements (HSEs), ethylene responsive elements (EREs), abscisic acid-responsive element (ABRE), MeJA responsive motifs (CGTCA-motif), drought stress and pathogen-responsive TC-rich repeat, and MYB binding sites (MBS). All of those cis-elements are located upstream of the TATA box (Figure 2). 


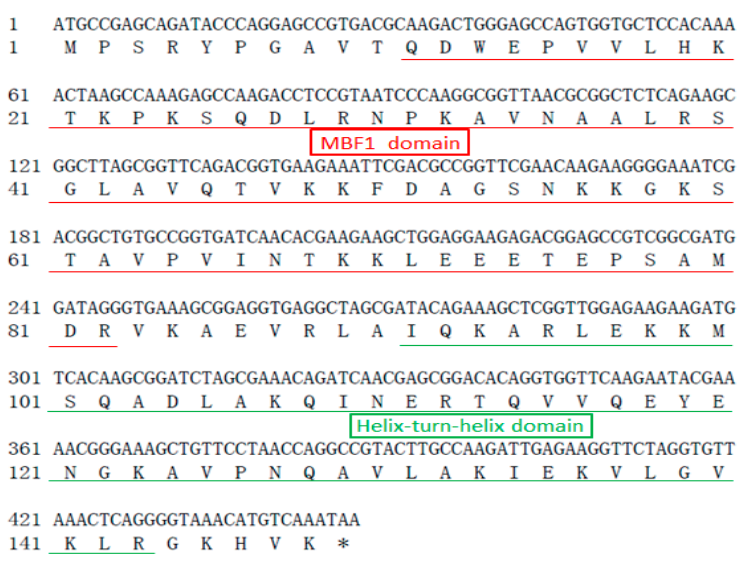

(a)

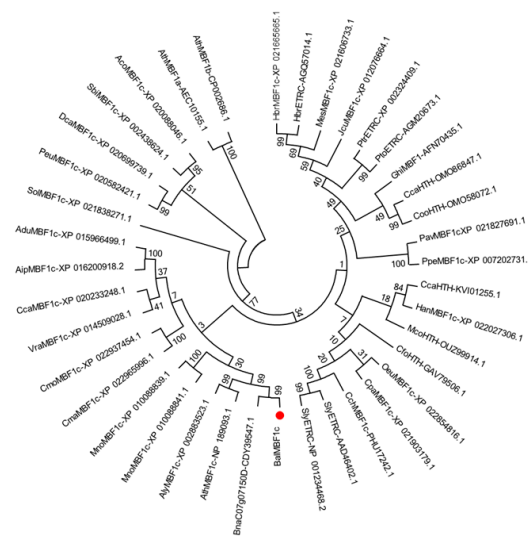

(b)

Figure 1. Sequence feature of BocMBF1c protein. (a) DNA and amino acid sequence of BocMBF1c. Full-length DNA and deduced amino acid sequence of BocMBF1c. Red underline indicates multi-protein bridging factor1 (MBF1) gene family domain, and green underline signatures helix-turn-helix (HTH) domain. (b) Phylogenetic tree constructed by the neighbor-joining method based on the MBF1c amino acid sequences.

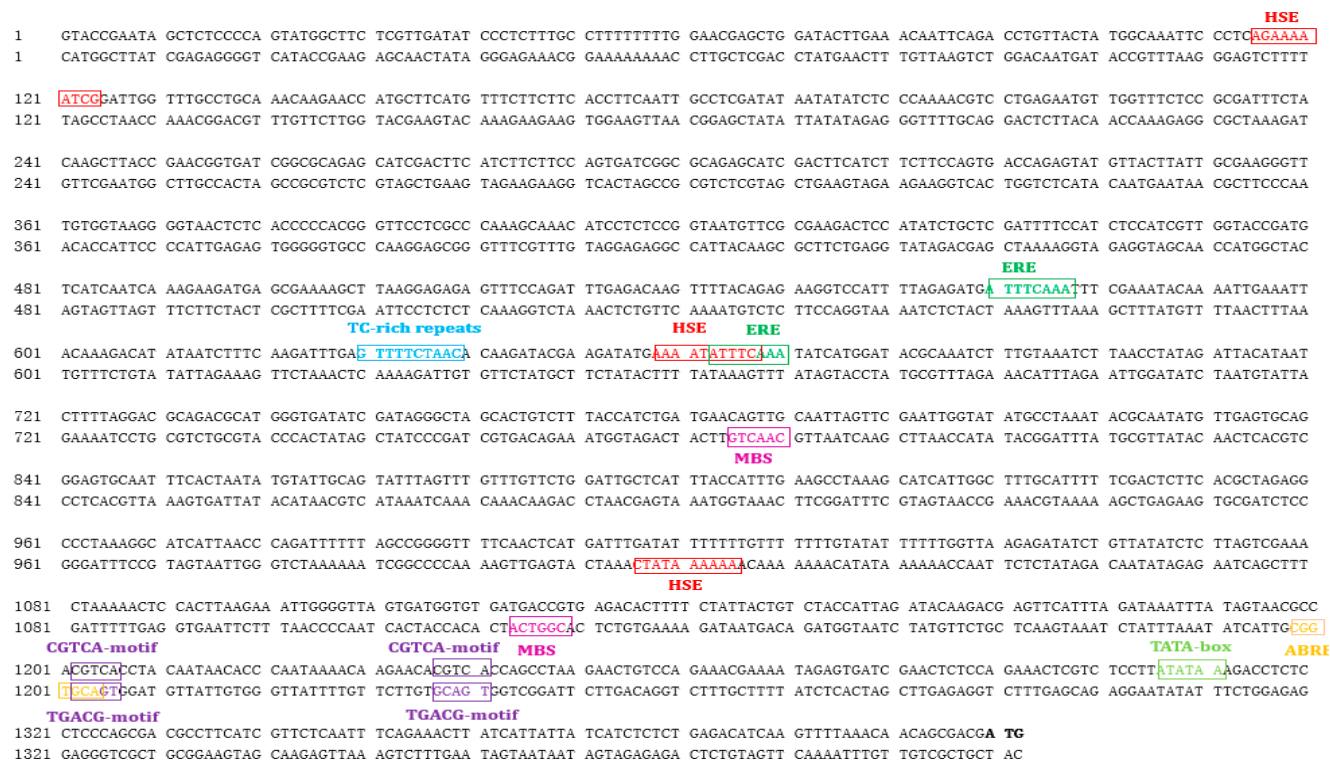

Figure 2. $1409 \mathrm{bp}$ upstream of the start code are shown as the BocMBF1c promoter. DNA elements in the BocMBF1c promoter were designated. The start code is designated as bold and the TATA box as grass green. Three heat shock elements (HSEs) are boxed in red, ethylene-responsive elements (EREs) in green boxes, ABA-responsive elements (ABREs) in yellow boxes, methyl jasmonate (MeJA)-responding elements in purple, MYB binding sites (MBS) in the purple red box, and TC-rich repeats as stress-responding elements in the blue box.

We cloned the coding region of BocMBF1c from genomic DNA, and the deduced BocMBF1c protein contained the MBF1 gene family domain and HTH domain (Figure 1a and Figure S2). The amino acid sequence of BocMBF1c shared 99\% and 94\% similarity with its ortholog from Brassica napus (CDY39547.1) and Arabidopsis, respectively (NP_189093.1). We queried the BocMBF1 protein sequence using the BLASTP program (https://blast.ncbi.nlm.nih.gov/Blast.cgi), and the resulting sequences all belonged to angiosperm. The phylogenetic tree showed BocMBF1c was clustered in one group with $M B F 1 c$ genes from dicots, while all MBF1c genes from monocots belonged to another group. In addition, BocMBF1c belonged to the MBF1c gene clade from the Brassicaceae family including Brassica 
napus and Arabidopsis. The AtMBF1a/b were clustered in an individual cluster, implying they belonged to different members of the MBF1 gene family (Figure 1b, Table S1).

\subsection{Transient Expression Analysis Showed BocMBF1c Promoter Activity Can Be Induced by Heat Stress}

The BocMBF1c promoter contains several HSEs, indicating its role in heat stress response. Thus, we analyzed the activity of the BocMBF1c promoter under heat treatment. We combined the $1409 \mathrm{bp}$ region upstream of the BocMBF1c start code with the CDS of green fluorescent protein (GFP), and then transiently expressed in Nicotiana ( $N$.) benthamiana leaf tissue by agroinfiltration under ambient and heat-stressed conditions. As predicted, the activity of the BocMBF1c promoter was rapidly stimulated under heat stress in Tabaco leaves (Figure 3a,b), which is consistent with previous reports [13].

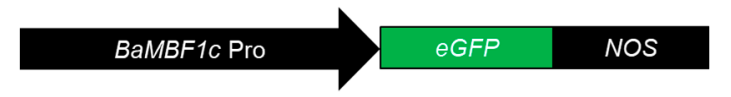

(a)

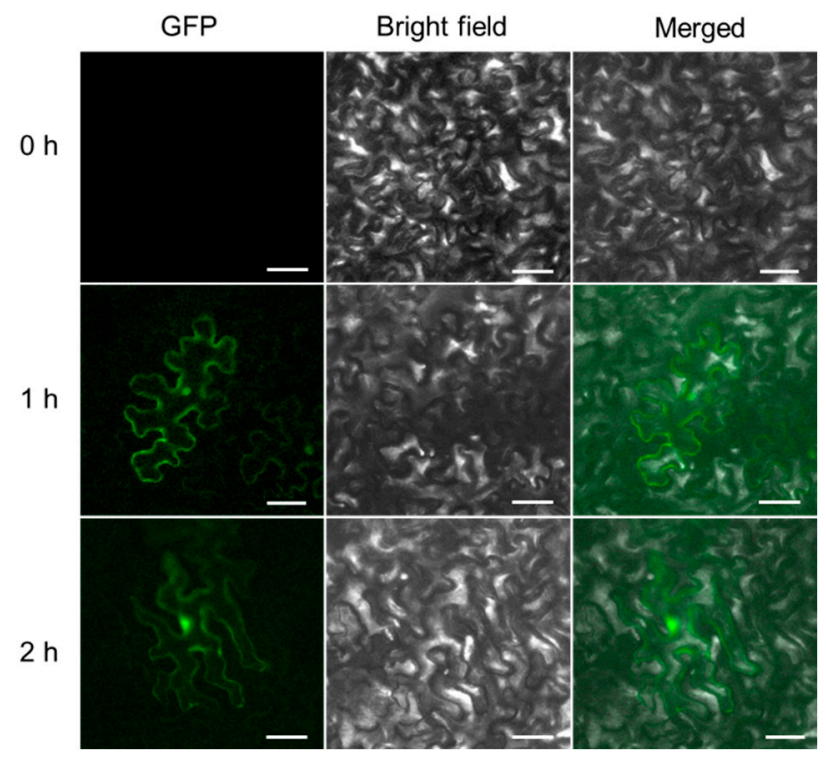

(b)

Figure 3. Heat treatment can stimulate BocMBF1c promoter activity. (a) Legend of pBI101-pBocMBF1c-GFP construct; BocMBF1c promoter was fused with green fluorescent protein (GFP) coding sequence (CDS) with the NOS terminator. (b) Treatment at $37^{\circ} \mathrm{C}$ induced increased expression in the Nicotiana (N.) benthamiana leaves after agroinfiltration. Scale bar, $50 \mu \mathrm{m}$.

\subsection{Expression Pattern of BocMBF1c}

Under a standard growth environment, expression of BocMBF1c was measured in various tissues of Chinese kale (Figure 4a). The results showed BocMBF1c had a comparatively higher expression level in combining sites (CS) and leaf veins (LV) and maintained a comparatively low expression level in other tissues (Figure $4 \mathrm{~b}$ ).

Under heat stress, BocMBF1c expression rapidly increased $>250$ fold within $0.5 \mathrm{~h}$ and maintained this high expression level for at least $8 \mathrm{~h}$ in the leaves of Chinese kale (Figure $5 \mathrm{a}$ ). When the Chinese kale was exposed to chilling conditions, BocMBF1c transcript abundance increased five-fold within $0.5 \mathrm{~h}$, then slowly returned to unstressed levels by $4 \mathrm{~h}$ post-treatment (Figure $5 \mathrm{~b}$ ). As illustrated in Figure 2, the BocMBF1c promoter possessed a series of hormone and other related cis-elements. The qPCR analysis confirmed that BocMBF1c was moderately responsive to salinity treatment and several plant hormones including MeJA, ABA, SA, and ET (Figure $5 c, d$ ). Interestingly, the temporal response patterns of BocMBF1c expression were distinct among different treatments. Under heat and cold stress, the increase in the BocMBF1c gene reached full extent within $0.5 \mathrm{~h}$. For salinity treatment, 
the expression level of BocMBF1c reached the maximum after $8 \mathrm{~h}$. When MeJA, ABA, SA, and ethephon (CEPA) were applied, the highest BocMBF1c transcription activities were detected after $2 \mathrm{~h}, 8 \mathrm{~h}, 2 \mathrm{~h}$, and $16 \mathrm{~h}$, respectively. Overall, our results indicated that other abiotic factors could also influence the transcript level of BocMBF1c besides responding to high-temperature conditions. These results indicate that BocMBF1c in Chinese kale involves resistance to multiple stresses including heat stress.

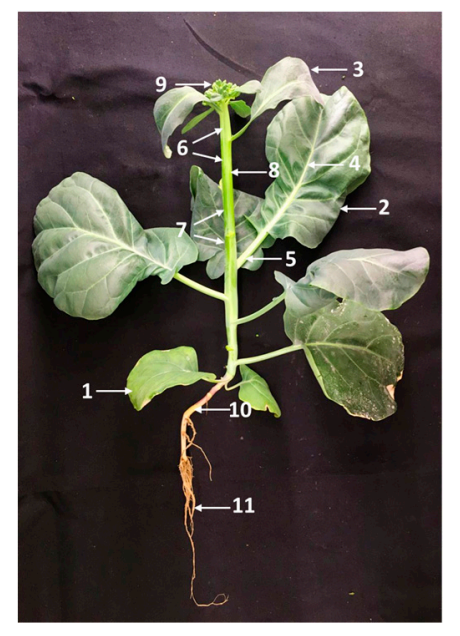

(a)

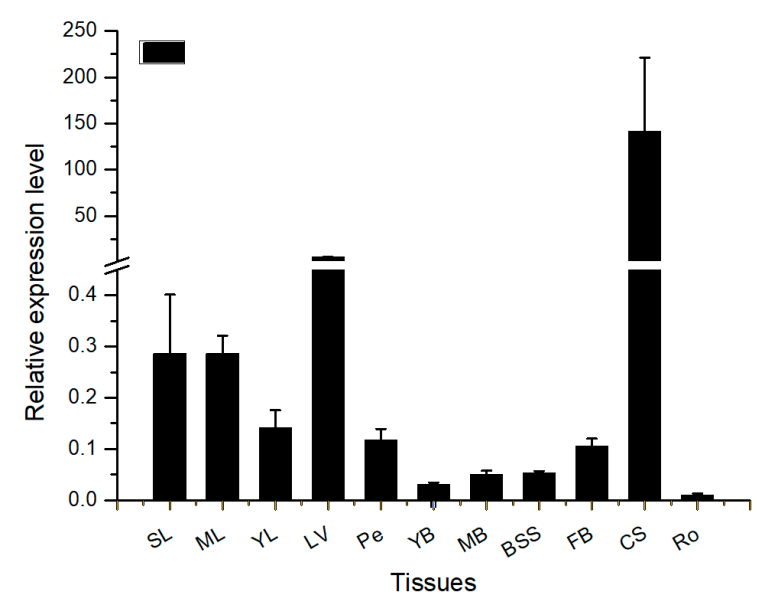

(b)

Figure 4. The expression pattern of BocMBF1c in different tissues under standard growth conditions. (a) Various tissues of Chinese kale. SL, senescent leaf; ML, mature leaf; YL, young leaf; LV, leaf vein; Pe, petiole; $\mathrm{YB}$, young bolting stem flesh; $\mathrm{MB}$, middle bolting stem flesh; BSS, bolting stem skin; FB, flower buds; CS, combining sites between stem and root; Ro, Roots. (b) Bolting stage tissues were harvested for expression specificity analysis. Quantitative PCR (qPCR) was used to measure BocMBF1c expression in different tissues, with the BocTubulin8 gene as the control. Data shown are the mean \pm SD of three biological replicates.

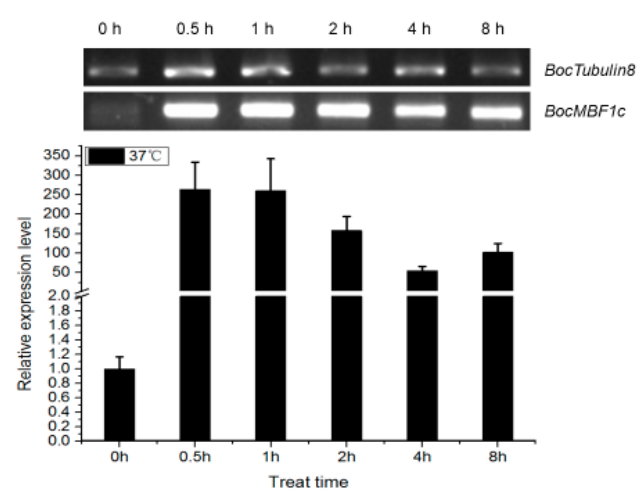

(a)

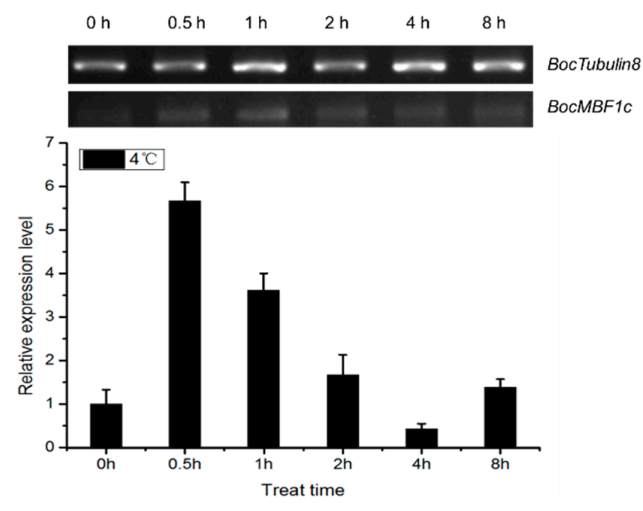

(b)

Figure 5. Cont. 


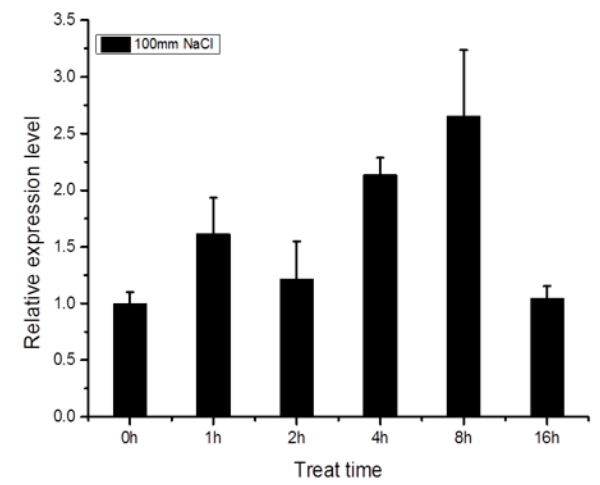

(c)

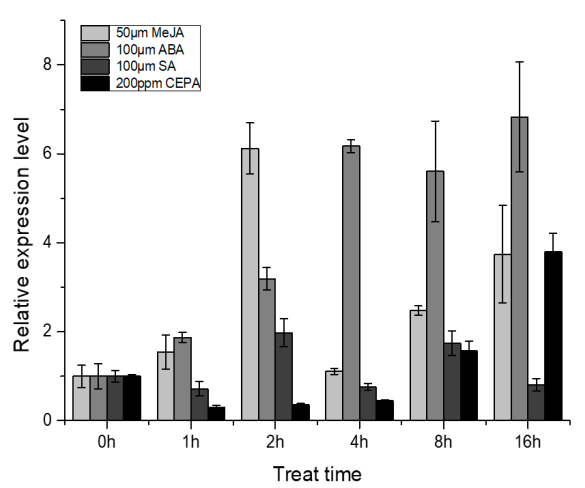

(d)

Figure 5. Responses of BocMBF1c to various abiotic treatments. (a) semiRT-PCR (reverse transcription PCR) and $\mathrm{qPCR}$ were used to determine the expression profile under heat stress $\left(37^{\circ} \mathrm{C}\right)$ in the leaf tissue of Chinese kale. (b) Expression of BocMBF1c under cold treatment $\left(4^{\circ} \mathrm{C}\right)$. semiRT-PCR and qPCR were used to determine the expression profile under cold condition in the leaf tissue of Chinese Kale. (c) Responses of BocMBF1c to salinity treatment. (d) Expression of BocMBF1c after applying hormones. All analyses used Tubulin 8 as an internal reference gene, and all data shown were normalized to mock treatments. Data shown are the mean \pm SD of three biological replicates.

\subsection{BocMBF1c Protein Localizes to the Nucleus under Heat Stress}

Under normal temperature, the GFP-tagged BocMBF1c protein shows no obvious distribution preference when transiently expressed in $N$. benthamiana leaf tissue (Figure $6 \mathrm{~b}$ ). However, the chimeric BocMBF1c-eGFP protein quickly concentrated into the nucleus when exposed to $37^{\circ} \mathrm{C}$ for $1 \mathrm{~h}$ (Figure $6 \mathrm{c}$ ). This translocation is critical for the functionality of this heat responsive transcription coactivator.

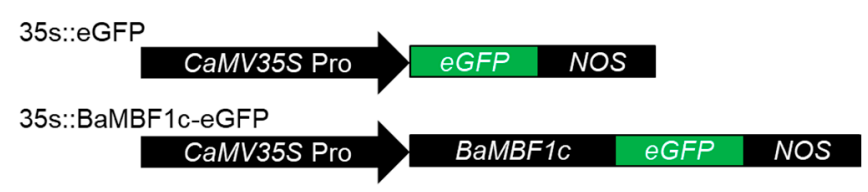

(a)

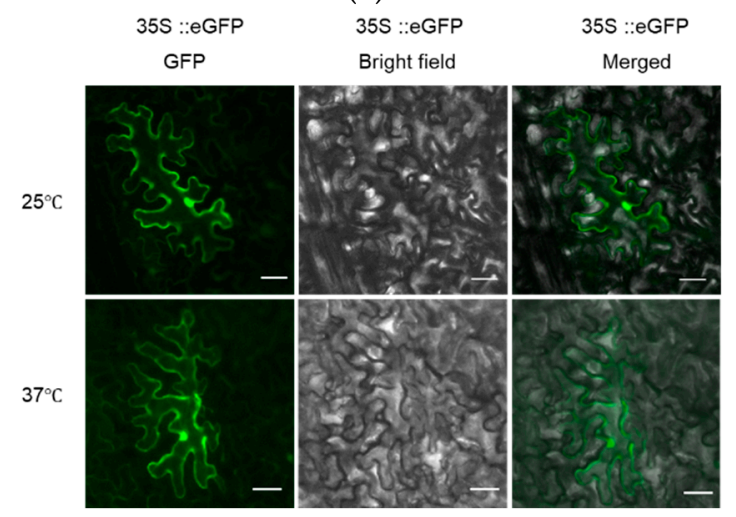

(b)

Figure 6. Cont. 


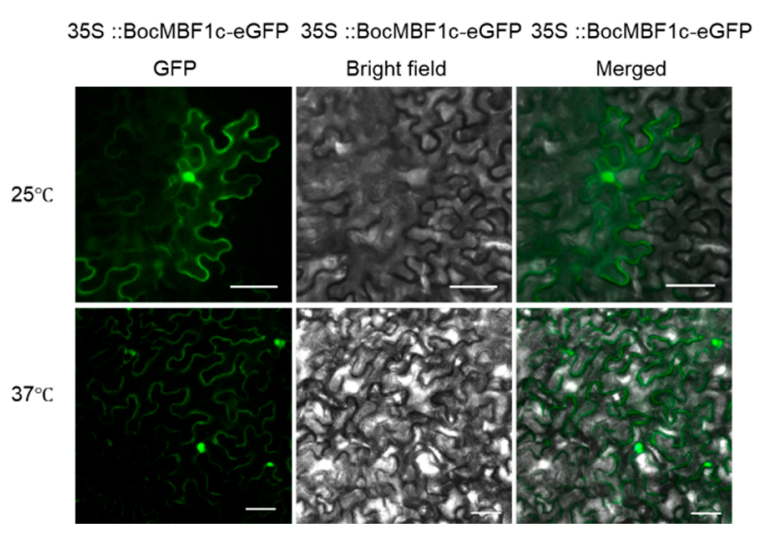

(c)

Figure 6. Heat stress led to the nucleus localization of the BocMBF1c protein. (a) Schematics of the constructs for 35s::eGFP and 35s::BocMBF1c-eGFP (pBE-BocMBF1c-eGFP). (b) The GFP protein alone has no obvious localization preference either under normal temperature or high temperature in $N$. benthamiana leaf tissue. (c) BocMBF1c translocated to the nucleus when exposed to $37^{\circ} \mathrm{C}$ for $1 \mathrm{~h}$. Scale bar, $50 \mu \mathrm{m}$.

\subsection{Phenotype and Thermotolerance Analysis of BocMBF1c Overexpression Lines}

We selected three $\mathrm{T}_{4}$ homozygous transgenic lines (Figure S3) overexpressed with the BocMBF1c and $\beta$-glucuronidase (GUS) fusion gene driven by the $35 \mathrm{~S}$ cauliflower mosaic virus (CaMV) promoter (Figure 7a) for further study. We named them BocMBF1c-OE1, BocMBF1c-OE2, and BocMBF1c-OE3. $\beta$-glucuronidase activity analyses were performed with 5 day old seedlings to validate the expression of BocMBF1c (Figure 7b). We also examined the growth of 2 week old BocMBF1c-OE plants. The BocMBF1c-OE plants show larger sizes compared to wild type (WT) under normal growth conditions (Figure 7c) which is consistent with a previous report [14]. The five-day-old seedlings grown on MS plates were used for thermal tolerance analysis. After treatment at $46^{\circ} \mathrm{C}$ for $2 \mathrm{~h}$, the transgenic lines showed a survival ratio of $21.7 \%$ to $30.0 \%$, while only $10.7 \%$ of the WT plants survived after heat stress (Figure 7d).

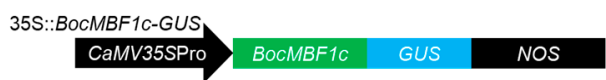

(a)

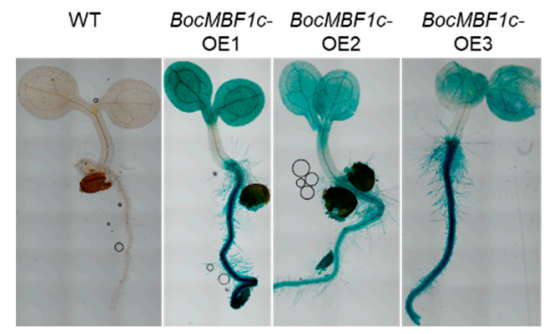

(b)

Figure 7. Cont. 


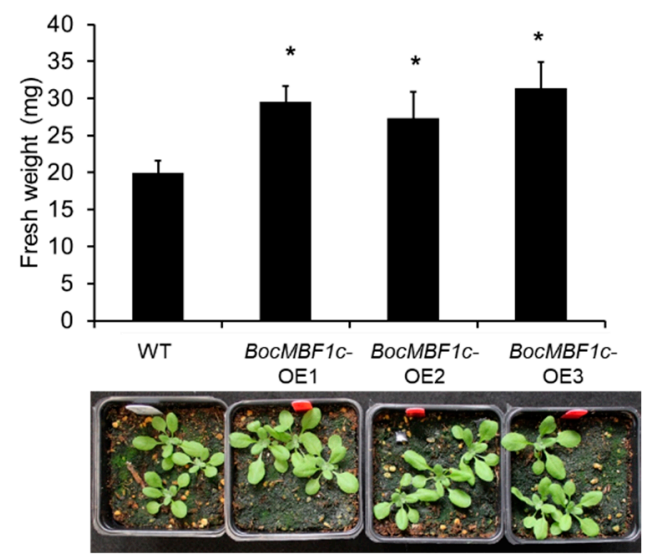

(c)

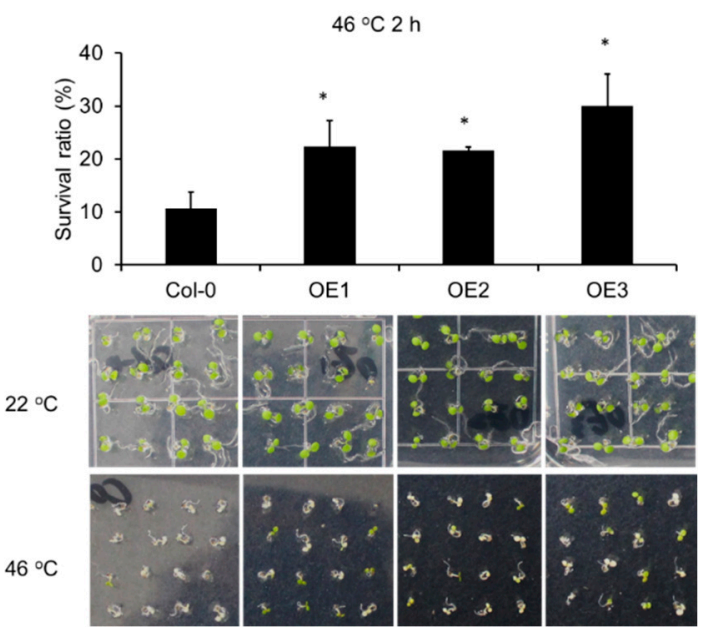

(d)

Figure 7. Phenotypic characterization of BocMBF1c-OE plants. (a) Schematic illustration of the 35s::BocMBF1c-GUS construct used for ectopic expression. (b) Expression of BocMBF1c was validated by $\beta$-glucuronidase (GUS) staining $(n \geq 15)$. (c) Compared with the wild type (WT) seedlings, the fresh weight of the BocMBF1c-OE plants indicated better growth, and the plants shown in the pictures were 14 days post-germination. Error bars represent the standard deviation of the means $(n=20)$. (d) All three BocMBF1c-OE lines showed a higher survival ratio after heat stress than WT $(n=100)$. Error bars are the mean \pm SD of three independent experiments. Photos were taken 2 days after heat stress. Asterisk indicates statistical significance by the Student's test $(p<0.05)$.

\section{Discussion}

\subsection{BocMBF1c Can Respond to Multiple Stresses and Hormone Treatment in Addition to Heat Stress in Chinese Kale}

We previously measured the effects of heat stress among 10 different Chinese kale varieties with different growth parameters, and the variety "Ai-jiao-xiang-gu" was found to be the most tolerable to heat stress [15]. We further measured the expression level of BocMBF1c; "Ai-jiao-xiang-gu" had the highest increase in expression level [15], indicating its important role in heat stress tolerance.

The promoter of BocMBF1c contained several HSEs; moreover, in N. benthamiana cells, the BocMBF1c promoter activity remained steady under ambient temperatures but was activated rapidly by high temperature stress. This is supported by semi-quantitative and qPCR evidence that the transcript abundance of BocMBF1c in planta is strongly and rapidly increased in response to heat stress treatment. Together, these data indicate that the expression of the BocMBF1c gene responds to heat stress via promoter activity. Our results showed that BocMBF1c can respond to cold, salinity, and several hormone treatments thus potentially possessing other functions besides thermal tolerance. 
Bioinformatic analysis predicted that the BocMBF1c promoter contained ERE- and ABRE-responding cis-elements, implying ERFs and ABFs (ABRE binding factors)/ABEB could regulate the expression of BocMBF1c. Accumulation of ABA can initiate the expression of stress-responsive genes and participate in the regulation of the network through ABFs/AREB [16]. Ethylene and JA can stimulate the expression of downstream stress-related genes by activating ERF1 [17]. Previous reports showed that AtMBF1c functions upstream of SA and ET during thermotolerance [10]. From our results, BocMBF1c reacted distinctly under different hormone treatments. The expression levels of BocMBF1c both increased after exogenous application of MeJA and ABA although with different temporal response feature. For MeJA and ABA treatment, BocMBF1c expression peaked after $2 \mathrm{~h}$ and $4 \mathrm{~h}$, respectively, which is possibly attributed to the difference in activation times for ERF1 and $\mathrm{ABFs} / \mathrm{AREB}$. After SA and ET application, the expression level of BocMBF1c both increased after a certain reduction period at the beginning. This indicates that BocMBF1c could possibly be negatively regulated by a high concentration of ET and SA, since the applied hormones would slowly degrade after application. With lower concentrations, ET can activate ERF1 thus stimulating the expression of BocMBF1c.

Overall, the accumulation of ABA, ET, and JA can activate corresponding transcription factors, which will then interact with the cis-elements located at the BocMBF1c promoter. The expressed BocMBF1c protein would then participate in the heat stress regulation network by regulating the downstream SA and ET signaling pathway (Figure 8).

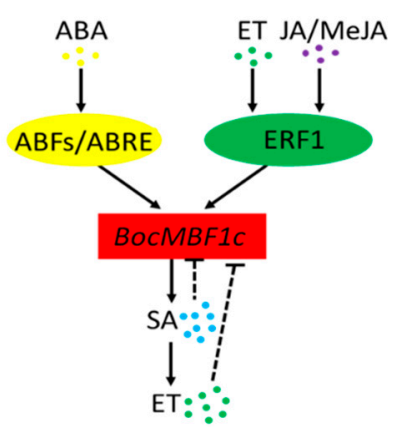

Figure 8. A model for the relation of BocMBF1c and other hormones. abscisic acid (ABA) and ethylene (ET) and jasmonic acid (JA)/MeJA activate ABRE binding factors (ABFs)/ABRE and Ethylene response factor1 (ERF1), respectively. ABFs/ABRE and ERF1 then upregulate the BocMBF1c gene by interacting with cis-elements in the promoter region. The BocMBF1c protein can regulate the salicylic acid (SA) and ethylene (ET) heat stress responsive pathway. High concentrations of SA and ET will inhibit the expression of BocMBF1c through negative feedback.

Through analysis of the Arabidopsis public database (http://bar.utoronto.ca/efp_arabidopsis/cgibin/efpweb.cgi), we found $A t M B F 1 c$ could be upregulated by heat, salt, and ABA treatment, and that it does not respond to cold and MeJA treatment (Figure S4) (http://bar.utoronto.ca/efp_arabidopsis/cgibin/efpWeb.cgi) [18]. The different responsive spectrums between BocMBF1c and AtMBF1c implies the $B o c M B F 1 c$ gene could participate in stress-related tolerance for the host plant.

The BocMBF1c protein quickly relocated to the nucleus when the cells were exposed to high temperature, further suggesting that this is a regulator for the expression of many heat stress-responsive genes. However, the mechanism of this nuclear translocation in a high-temperature environment remains unknown and should be the focus of future study. Likewise, downstream genes activated by this transcription factor remain unknown in Chinese kale, and transcriptome analysis by next-generation sequencing would be useful in the identification of candidate downstream genetic elements. Expression of these elements as well as known targets from other species can be further confirmed by qPCR. Earlier studies found the "CTAGA" element as a potential binding sequence for downstream genes; thus, it may be interesting to analyze the orthologs of the downstream genes in Chinese kale. This may 
reveal the reason for the relative temperature sensitivity of Chinese kale. Thus, it is highly likely that the BocMBF1c gene is necessary for multiple biotic/abiotic stresses in Chinese kale.

\subsection{BocMBF1c Can be Applied to Improve the Productivity of Chinese Kale}

The high-level conservation of MBF1c sequences (Table S1 and Figure S2) implies that this gene is critical for thermal tolerance and climate adaptation. For Chinese kale, bolting stems are the main edible tissue. The genetic mechanism underlying declined yield and quality of bolting stems under heat stress are still elusive in Chinese kale; cloning of BocMBF1c genes as a key heat tolerance factor would shed light on improving the heat tolerance of Chinese kale.

Transformation of Chinese kale is readily feasible and has been used for improving drought stress tolerance [19]. Our results indicate that the BocMBF1c gene can respond to heat treatment from both transcription and protein localization level. Overexpression BocMBF1c in Arabidopsis result in larger-sized plants with enhanced heat tolerance, while knockout of the BocMBF1c gene will be needed to fully validate its role in the thermal tolerance of Chinese kale, for example, by the CRISPR/Cas9-based gene editing technique [20]. Ectopic expression of AtMBF1c can improve tolerance to bacterial infection, heat, and osmotic stress in Arabidopsis [14]. Further study showed that overexpression of AtMBF1c could improve thermal tolerance without impairing the yield in Arabidopsis [10], soybean [11], and rice [21] under controlled conditions. A recent report showed that overexpression of MBF1c in Antarctic moss could make the plant bigger and enhance tolerance to salinity stress in Arabidopsis [12]. Thus, it will be intriguing to overexpress the BocMBF1c gene as a potential solution to improving the biotic/abiotic stress resistance and productivity of Chinese kale.

\section{Materials and Methods}

\subsection{Plant Growth and Abiotic Treatment Conditions}

We collected Chinese kale samples for tissue-specific expression analysis from the greenhouse $\left(22-25^{\circ} \mathrm{C}\right)$ at the South China Agricultural University (Guangzhou, China) [2]. For abiotic treatment, Chinese kale (B. oleracea var. chinensis Lei) variety "Ai-jiao-xiang-gu" were grown in a culture room under controlled conditions at $25^{\circ} \mathrm{C}$, with a $16 \mathrm{~h}$ light $/ 8 \mathrm{~h}$ dark cycle. Chinese kale seedlings of $4 \sim 6$ true leaves were transferred to the growth chamber for $24 \mathrm{~h}$ with the same conditions, and then the temperature was switched to heat $\left(37^{\circ} \mathrm{C}\right)$ and cold $\left(4^{\circ} \mathrm{C}\right)$ treatments. Leaf tissues were harvested for RNA extraction at $0 \mathrm{~h}, 0.5 \mathrm{~h}, 1 \mathrm{~h}, 2 \mathrm{~h}, 4 \mathrm{~h}$, and $8 \mathrm{~h}$.

For salinity treatment, each Chinese kale seedling of $4 \sim 6$ true leaves were sprayed and watered by $100 \mathrm{~mL}$ of $100 \mathrm{mM} \mathrm{NaCl}$, and leaf tissues were collected with the timeline of $0 \mathrm{~h}, 1 \mathrm{~h}, 2 \mathrm{~h}, 4 \mathrm{~h}, 8 \mathrm{~h}$, and $16 \mathrm{~h}$. Similarly, hormone treatments were carried out by applying $50 \mu \mathrm{M}$ of MeJA solution, $100 \mu \mathrm{M}$ ABA, $100 \mu \mathrm{M} \mathrm{SA}$, and $100 \mathrm{ppm}$ CEPA, respectively, and leaf tissues were collected along the same timeline. All samples used in this study were collected from three separated Chinese kale.

Arabidopsis thaliana (ecotype Columbia) were grown in chamber under controlled condition at $22{ }^{\circ} \mathrm{C}$ day $/ 18^{\circ} \mathrm{C}$ night, $70 \%$ relative humidity, and $100 \mu \mathrm{mol} \mathrm{m}{ }^{-2} \mathrm{~s}^{-1}$ with a $16 \mathrm{~h}$ light $/ 8 \mathrm{~h}$ dark light cycle. Arabidopsis seeds were germinated on MS plates or soil (Sunshine ${ }^{\circledR}$ Mix \#8, Sun Gro Horticulture) after being sterilized with $10 \%$ bleach for $10 \mathrm{~min}$. All seeds were kept at $4{ }^{\circ} \mathrm{C}$ for at least 2 days before transfer to the growing chamber.

\subsection{Cloning of the BocMBF1c CDS and Promoter}

Leaves of Chinese kale were harvested for genomic DNA extraction [22]. We used the MBF1c gene from Brassica rapa (LOC103828628) as a reference sequence (Supplementary B1) to design primers for the CDS of BocMBF1c. The gDNA fragment containing the BocMBF1c CDS was amplified with primer MBF1c-up (5'-TCGAACTCTCCAGAAACTCGT-3') and MBF1c-dw (5'-CGTTTGCCAGATACGATGT-3'). Next, BocMBF1c promoter was amplified with primer MBF1cP-F (5'-AGAATCCCCATTGACAGCCT-3') and MBF1cP-R (5'-GGCATCGTCGCTGTTGTTTA-3'), designed 
from the gene sequence of Brassica oleracea (Supplementary B2). All PCRs were carried out with HiFiTaq PCR StarMix (GeneStar) according to the manufacturer's protocol.

\subsection{Sequence Analysis of the BocMBF1c Gene}

The open reading frame (ORF) of BocMBF1c was predicted by the ORF finder (http:// www.bioinformatics.org/sms2/orf_find.html), and the amino acid sequence was generated by the Expasy-Translate tool (https://web.expasy.org/translate/). Sequence query and alignment were carried out with BLAST (https://blast.ncbi.nlm.nih.gov/Blast.cgi). A phylogenetic tree was constructed with the software Mega 6.05, and the conserved sites were identified by DNAMEN.

The cis-elements of the BocMBF1c promoter were predicted by PlantCARE Database (http: //bioinformatics.psb.ugent.be/webtools/plantcare/html/).

\subsection{Promoter Activity and Subcellular Localization}

The putative BocMBF1c promoter of the $1409 \mathrm{bp}$ upstream sequence from the initiation codon was cloned into the binary vector pBI101-GFP with In-Fusion cloning, using primers designed following the manufacturer's instructions (http://bioinfo.clontech.com/infusion/convertPcrPrimersInit. do), including pBI101 infusion M-P-F (5'-GCAGGTCGACTCTAGAGGTACCGAATAGCTCTCCCC-3') and pBI101 infusion M-P-R (5'- CTTTACCCATTCTAGAGGCATCGTCGCTGTTGTTTA-3'). Similarly, CDS of the BocMBF1c was inserted into the pBE-eGFP construct by the In-Fusion technique, with primers MBF1-yxb-F (5'-CGCGGGCCCGGGATCCATGCCGAGCAGAT-3') and MBF1-yxb-R (5'-GGCGACCGGTGGATCCTTGACATGTTT-3'). Xba I was used to digest the pBI101-GFP vector, and pBE-eGFP was digested by BamH I, resulting in pBI101-pBocMBF1c-GFP and pBE-BocMBF1c-eGFP constructions.

Transient expression was carried out in the leaf tissue of Nicotiana benthamiana seedlings with 4 to 6 true leaves, and with Agrobacterium strain GV3101 harboring the pBI101-pBocMBF1c-GFP and pBE-BocMBF1c-eGFP constructs. The GFP signal was observed two days after agro-infiltration under fluorescent microscopy [23].

\subsection{Gene Expression Patterns of BocMBF1c by $q P C R$ and Semi-Quantitative RT-PCR}

The RNA was extracted using HiPure Plant RNA Kits (Magen), and HiScript ${ }^{\circledR}$ II Q Select RT SuperMix (Vazyme) was used for cDNA synthase. Gene expression was assessed by real-time PCR using AceQ ${ }^{\circledR}$ qPCR SYBR ${ }^{\circledR}$ Green Master Mix (Vazyme) and Bio-Rad iQ5 Multicolor Real-Time PCR Detection System. Primers MBF1c-YG1-UP (5'-GTTAACGCGGCTCTCAGAAG-3') and MBF1c YG1-DW (5'-TCCTCCAGCTTCTTCGTGTT-3') were used for BocMBF1c; Tubulin8 was used as an internal control with primers Tubulin-F (5'-CTTCTTTCGTGCTCATTTTGCC-3') and Tubulin-R (5'-CCATTCCCTCGTCTCCACTTCT-3') [19]. The qPCR conditions: initial denaturation of $95^{\circ} \mathrm{C}$ for $5 \mathrm{~min}$, then 40 cycles of $95^{\circ} \mathrm{C}$ for $10 \mathrm{~s}$ followed by $60^{\circ} \mathrm{C}$ for $30 \mathrm{~s}$; melting curves were obtained by gradually increasing the temperature from $60^{\circ} \mathrm{C}$ to $95^{\circ} \mathrm{C}$ for $15 \mathrm{~s}$ at a rate of $0.5^{\circ} \mathrm{C} / \mathrm{s}$. Relative expression was calculated by the $\Delta \Delta \mathrm{Ct}$ threshold method [24]. For heat and cold treatment, semi-quantitative RT-PCR was used to detect the expression pattern of BocMBF1c with Recombinant Taq DNA Polymerase TaKaRa Taq ${ }^{\mathrm{TM}}$ (Takara) and the same primers of qPCR. Semi-quantitative RT-PCR conditions: initial denaturation of $94{ }^{\circ} \mathrm{C}$ for $2 \mathrm{~min}$, then 26 cycles for Tubulin 8 and 30 cycles for BocMBF1c of $95^{\circ} \mathrm{C}$ for $30 \mathrm{~s}, 56^{\circ} \mathrm{C}$ for $15 \mathrm{~s}, 72^{\circ} \mathrm{C}$ for $10 \mathrm{~s}$. Three biological replicates with triple technical replicates were used for all samples.

\subsection{Arabidopsis Transformation and Phenotypic Analysis of Transgenic Plants}

The BocMBF1c coding sequence was fused with GUS and then expressed in Arabidopsis thaliana driven by the $35 S \mathrm{CaMV}$ promoter and then integrated into the $p B 1121$ binary vector. The floral dipping method [25] was used for Arabidopsis transformation. Transgenic seeds were selected on a 0.5 MS agar plate with $50 \mathrm{mg} / \mathrm{L}$ kanamycin and $300 \mathrm{mg} / \mathrm{L}$ timentin and then transplanted to soil for setting 
the seeds under standard growth conditions. Three homozygous transgenic lines from independent transformation events were used for further study (Figure S3).

Five-day-old BocMBF1c-OE and Col-0 Arabidopsis seedlings were collected for GUS staining analysis after germination on MS plates [13]. Two-week-old seedlings grown on soil were harvested for measuring fresh weight. For thermal tolerance analysis, five-day-old seedlings grown on MS plates were treated at $46^{\circ} \mathrm{C}$ for $2 \mathrm{~h}$, and then these plates were put back into growth chambers under normal condition for another 2 days.

Supplementary Materials: Supplementary materials can be found at http://www.mdpi.com/1422-0067/20/22/ 5637/s1.

Author Contributions: Conceptualization, C.C. and J.L.; Data curation, L.Z.; Formal analysis, L.Z., X.-L.M. and B.C.; Investigation, L.Z.; Methodology, L.Z., B.Y. and C.C.; Project administration, G.C. and J.L.; Supervision, J.L.; Validation, L.Z. and X.-L.M.; Visualization, L.Z. and X.-L.M.

Funding: This work was supported by the key project of Guangdong Science and Technology Section, grant numbers 2013B051000069 and 2014B020202005, and the key project of the Guangzhou Science Technology and Innovation Commission, grant number 201508030021.

Acknowledgments: We acknowledge Joanne Ernest (Global Institute for Food Security) for providing very valuable comments when preparing this manuscript; we thank Yehua He for providing the pBI-101-eGFP construct and Fengpin Wang for aiding in the agro-infiltration in tobacco leaves.

Conflicts of Interest: The authors declare no conflict of interest.

\section{Abbreviations}

$\begin{array}{ll}\text { MBF } & \text { Multi-protein bridging factor } \\ \text { HTH } & \text { Helix-turn-helix } \\ \text { HSEs } & \text { Heat shock elements } \\ \text { GFP } & \text { Green fluorescent protein } \\ \text { eGFP } & \text { Enhanced green fluorescent protein } \\ \text { ABA } & \text { Abscisic acid } \\ \text { ABRFs } & \text { ABA responsive transcription factors } \\ \text { HSF } & \text { Heat stress transcription factor } \\ \text { HSPs } & \text { Heat shock proteins } \\ \text { MeJA } & \text { Methyl jasmonate } \\ \text { SA } & \text { Salicylic acid } \\ \text { ET } & \text { Ethylene } \\ \text { JA } & \text { Jasmonic acid } \\ \text { ERF } & \text { Ethylene response factor } \\ \text { DRE } & \text { Dehydration-responsive element } \\ \text { ORF } & \text { Open reading frame } \\ \text { CDS } & \text { Coding sequence } \\ \text { ERE } & \text { Ethylene-responsive elements } \\ \text { ABRE } & \text { Abscisic acid-responsive element } \\ \text { MBS } & \text { MYB binding site } \\ \text { N. } & \text { Nicotiana } \\ \text { qPCR } & \text { Quantitative PCR } \\ \text { CEPA } & \text { Ethephon } \\ \text { RT-PCR } & \text { Reverse transcription PCR } \\ \text { GUS } & \text { ß-Glucuronidase } \\ \text { CaMV } & \text { Cauliflower mosaic virus } \\ \text { WT } & \text { Wild type } \\ \text { ABFs } & \text { ABRE binding factors } \\ & \end{array}$




\section{References}

1. Lei, J.; Chen, G.; Chen, C.; Cao, B. Germplasm Diversity of Chinese Kale in China. Hortic. Plant J. 2017, 3 , 101-104. [CrossRef]

2. Wu, S.; Lei, J.; Chen, G.; Chen, H.; Cao, B.; Chen, C. De novo Transcriptome Assembly of Chinese Kale and Global Expression Analysis of Genes Involved in Glucosinolate Metabolism in Multiple Tissues. Front. Plant Sci. 2017, 8, 92. [CrossRef] [PubMed]

3. Si, Y.; Chen, G.; Lei, J.; Cao, B.; Feng, E. Analysis on Composition and Content of Glucosinolates in Different Genotypes of Chinese Kale. China Veg. 2009, 6, 7-13.

4. Yang, X.; Yang, Y. The Effects of Temperature on Flower Bud Differentiation, Yield and Quality Formation in Chinese Kale (Brassica alboglabra Bailey). J. S. China Agric. Univ. 2002, 23, 5-7.

5. Bita, C.E.; Gerats, T. Plant tolerance to high temperature in a changing environment: Scientific fundamentals and production of heat stress-tolerant crops. Front. Plant Sci. 2013, 4, 273. [CrossRef] [PubMed]

6. Wang, X.; Zhuang, L.; Shi, Y.; Huang, B. Up-Regulation of HSFA2c and HSPs by ABA Contributing to Improved Heat Tolerance in Tall Fescue and Arabidopsis. Int. J. Mol. Sci. 2017, 18, 1981. [CrossRef] [PubMed]

7. Duan, Y.-H.; Guo, J.; Ding, K.; Wang, S.-J.; Zhang, H.; Dai, X.-W.; Chen, Y.-Y.; Govers, F.; Huang, L.-L.; Kang, Z.-S. Characterization of a wheat HSP70 gene and its expression in response to stripe rust infection and abiotic stresses. Mol. Biol. Rep. 2011, 38, 301-307. [CrossRef] [PubMed]

8. Khan, M.I.R.; Iqbal, N.; Masood, A.; Per, T.S.; Khan, N.A. Salicylic acid alleviates adverse effects of heat stress on photosynthesis through changes in proline production and ethylene formation. Plant Signal. Behav. 2013, 8, e26374. [CrossRef] [PubMed]

9. Qu, A.-L.; Ding, Y.-F.; Jiang, Q.; Zhu, C. Molecular mechanisms of the plant heat stress response. Biochem. Biophys. Res. Commun. 2013, 432, 203-207. [CrossRef] [PubMed]

10. Suzuki, N.; Bajad, S.; Shuman, J.; Shulaev, V.; Mittler, R. The transcriptional co-activator MBF1c is a key regulator of thermotolerance in Arabidopsis thaliana. J. Biol. Chem. 2008, 283, 9269-9275. [CrossRef] [PubMed]

11. Suzuki, N.; Sejima, H.; Tam, R.; Schlauch, K.; Mittler, R. Identification of the MBF1 heat-response regulon of Arabidopsis thaliana. Plant J. 2011, 66, 844-851. [CrossRef] [PubMed]

12. Alavilli, H.; Lee, H.; Park, M.; Lee, B. Antarctic Moss Multiprotein Bridging Factor 1c Overexpression in Arabidopsis Resulted in Enhanced Tolerance to Salt Stress. Front. Plant Sci. 2017, 8, 1206. [CrossRef] [PubMed]

13. Tsuda, K.; Yamazaki, K.I. Structure and expression analysis of three subtypes of Arabidopsis MBF1 genes. Biochim. Biophys. Acta-Gene Struct. Expr. 2004, 1680, 1-10. [CrossRef] [PubMed]

14. Suzuki, N. Enhanced Tolerance to Environmental Stress in Transgenic Plants Expressing the Transcriptional Coactivator Multiprotein Bridging Factor 1c. Plant Physiol. 2005, 139, 1313-1322. [CrossRef] [PubMed]

15. Wang, H.; Chen, G.; Chen, C.; Cao, B.; Zou, L.; Lei, J. Identification of Heat Tolerance in Chinese Kale and the Expression Analysis of Heat Tolerance Transcription Factor MBF1c. China Veg. 2017, 2, 30-37.

16. Jacob, P.; Hirt, H.; Bendahmane, A. The heat-shock protein/chaperone network and multiple stress resistance. Plant Biotechnol. J. 2017, 15, 405-414. [CrossRef] [PubMed]

17. Cheng, M.-C.; Liao, P.-M.; Kuo, W.-W.; Lin, T.-P. The Arabidopsis ETHYLENE RESPONSE FACTOR1 Regulates Abiotic Stress-Responsive Gene Expression by Binding to Different cis-Acting Elements in Response to Different Stress Signals. Plant Physiol. 2013, 162, 1566-1582. [CrossRef] [PubMed]

18. Toufighi, K.; Brady, S.M.; Austin, R.; Ly, E.; Provart, N.J. The botany array resource: E-Northerns, expression angling, and promoter analyses. Plant J. 2005, 43, 153-163. [CrossRef] [PubMed]

19. Zhu, Z.; Sun, B.; Xu, X.; Chen, H.; Zou, L.; Chen, G.; Cao, B.; Chen, C.; Lei, J. Overexpression of AtEDT1/HDG11 in Chinese Kale (Brassica oleracea var. alboglabra) Enhances Drought and Osmotic Stress Tolerance. Front. Plant Sci. 2016, 7, 1285. [CrossRef] [PubMed]

20. Ma, X.; Zhang, Q.; Zhu, Q.; Liu, W.; Chen, Y.; Qiu, R.; Wang, B.; Yang, Z.; Li, H.; Lin, Y.; et al. A Robust CRISPR/Cas9 System for Convenient, High-Efficiency Multiplex Genome Editing in Monocot and Dicot Plants. Mol. Plant 2015, 8, 1274-1284. [CrossRef] [PubMed]

21. Qin, D.; Wang, F.; Geng, X.; Zhang, L.; Yao, Y.; Ni, Z.; Peng, H.; Sun, Q. Overexpression of heat stress-responsive TaMBF1c, a wheat (Triticum aestivum L.) Multiprotein Bridging Factor, confers heat tolerance in both yeast and rice. Plant Mol. Biol. 2015, 87, 31-45. [CrossRef] [PubMed] 
22. Doyle, J.J.; Doyle, J.L. A rapid DNA isolation procedure for small quantities of fresh leaf tissue. Phytochem. Bull. 1987, 19, 11-15.

23. Goodin, M.M.; Zaitlin, D.; Naidu, R.A.; Lommel, S.A. Nicotiana benthamiana: Its History and Future as a Model for Plant-Pathogen Interactions. Mol. Plant-Microbe Interact. 2008, 21, 1015-1026. [CrossRef] [PubMed]

24. Livak, K.J.; Schmittgen, T.D. Analysis of relative gene expression data using real-time quantitative PCR and. Methods 2001, 25, 402-408. [CrossRef] [PubMed]

25. Clough, S.J.; Bent, A.F. Floral dip: A simplified method for Agrobacterium-mediated transformation of Arabidopsis thaliana. Plant J. 1998, 16, 735-743. [CrossRef] [PubMed] 